\title{
Educating primary healthcare providers about kidney disease
}

Aminu K. Bello (10 ' and David W. Johnson (10) ${ }^{2,3,4 \times}$

Chronic kidney disease is a major public health problem that is associated with excessive morbidity, mortality and healthcare costs. However, limited clinician awareness of chronic kidney disease is universally identified as a key barrier to care. A concerted effort is urgently needed to address the knowledge gaps of primary care providers.

estimates
suggest that
about $90 \%$ of
[people with
CKD] do not
know that
they have the
disease

'Department of Medicine, University of Alberta, Edmonton, Alberta, Canada.

${ }^{2}$ Translational Research Institute, Brisbane, Queensland, Australia.

${ }^{3}$ Department of Nephrology, Princess Alexandra Hospital, Brisbane, Queensland, Australia.

${ }^{4}$ Australasian Kidney Trials Network, University of Queensland, Brisbane Queensland, Australia.

凶e-mail:david.johnson2@ health.qld.gov.au

https://doi.org/10.1038/ s41581-021-00527-y
Chronic kidney disease (CKD) affects 850 million people worldwide and disproportionately burdens vulnerable and disadvantaged populations in low- and middle-income countries ${ }^{1}$. This devastating illness spans the entire life course and is associated with increased morbidity, mortality and healthcare costs, as well as a negative effect on quality of life ${ }^{2}$. In 2017, CKD contributed directly or indirectly to $4.6 \%$ of global deaths ${ }^{3}$, a figure that has steadily increased over time. The prevalence of CKD is expected to rise even further in the aftermath of the COVID-19 pandemic as this disease is associated with an increased long-term risk of adverse kidney outcomes.

Although CKD is common and treatable, estimates suggest that about $90 \%$ of affected people do not know that they have the disease. A key reason for this lack of awareness is the limited capacity of the healthcare workforce to identify and appropriately manage CKD, particularly in primary care settings, owing to a lack of availability of healthcare workers as well as limited knowledge and skills. In 2017, the International Society of Nephrology Global Kidney Health Atlas survey of 125 countries (representing an estimated $93 \%$ of the world's population) found that the top barriers to optimal kidney care were patient-related factors (knowledge and attitude; $91 \%$ of responding countries) and non-nephrologist physician-related factors (availability, access, knowledge and attitude; $84 \%$ of responding countries) $)^{5}$. Almost two-thirds (64\%) of countries rated CKD awareness amongst primary care providers as low or extremely low, and this awareness was more likely to be extremely low in low-income countries.

In an attempt to overcome these challenges, the theme for World Kidney Day 2022 is "Kidney health for all. Bridge the knowledge gap to better care". As the overwhelming majority of patients with CKD are predominantly managed in the primary healthcare system without seeing a nephrologist, it is of paramount importance to address the knowledge gaps of primary healthcare providers, including general practitioners, family physicians, nurses, community pharmacists and
Indigenous healthcare workers. The onus is on nephrology societies to work in partnership with allied health organizations, nurse associations, kidney-health professional working groups, and other related consumer organizations and primary health networks to ensure the embedding of optimal kidney care in the curricula of medical, nursing and other allied health training programmes across the globe.

It is essential to provide access to comprehensive kidney-care education programmes for all primary healthcare providers, which includes all care-givers engaged in providing health promotion, disease prevention and treatment ${ }^{6}$. Such programmes should be targeted, informed by analyses of local learning needs, goal-oriented with measurable objectives, appropriate to the local culture and resource setting, and multi-pronged, including interactive web-based sessions (webinars), face-to-face training (workshops and seminars), case-based discussions, conference symposia and online learning modules. Participation in these educational programmes should be incentivized by professional societies or colleges through the conferring of continuing medical education credits. Nephrology societies should also advocate vigorously for governments to implement incentive payments for the performance of kidney health checks (that is, measurement of blood pressure, estimated glomerular filtration rate and urine albumin:creatinine ratio) as part of integrated health checks and annual primary care screenings for patients who are at risk of kidney disease, including those with diabetes mellitus and/or hypertension.

The key objectives of any education programme for primary healthcare providers should underscore the urgent public health threat of CKD and its strong links with other chronic diseases (for example, diabetes mellitus, hypertension and cardiovascular disease); highlight CKD risk factors that should trigger the performance of a kidney health check; include how to perform and interpret a kidney health check; inform how to stratify kidney and cardiovascular risk and link each risk stage with a specific targeted clinical action plan (including lifestyle 


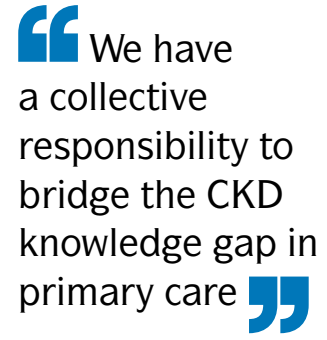

modification and medical interventions); and provide guidance regarding practice organizational approaches to CKD management, care of common issues in CKD, when to refer to a nephrologist, medication considerations and advance care planning. Education programmes can be reinforced by providing primary healthcare workers with access to specific primary healthcare guidelines (such as CKD handbooks and online care pathways), decision-support software tools ${ }^{7}$ and freely available mobile phone applications, such as CKD-GO! (Kidney Health Australia, Adelaide), that enable healthcare providers and patients to develop personalized action plans on the basis of simple kidney measures such as estimated glomerular filtration rate and urine albumin: creatinine ratio.

In many parts of the world, particularly in lowincome countries with under-resourced health systems and disproportionately large disease burdens, healthcare delivery, structures and training are often the product of established tradition rather than of evidence and are often incongruent with health-system changes and demands $s^{6,8}$. It is therefore crucially important to evaluate the effect of knowledge transfer though rigorous evaluations. Implementation of acquired knowledge can be considerably amplified by facilitated quality improvement and quality assurance activities based on well-tested evaluation frameworks such as 'Plan, Do, Study and Act' cycles or other culture- and context-specific frameworks. An apt example is the Educating for Equity Care Framework, which is designed to train culturally adept healthcare providers to help patients with diabetes to set individualized goals and to provide self-management support for these patients ${ }^{9}$. The educational framework must ensure that practitioners are equipped with the tools and skills to conduct audits of their approach to CKD in the community. Other innovative and effective strategies include primary care amplification or 'beacon' models, in which interested practitioners receive training from specialists within a beacon primary care practice. This approach integrates chronic disease management into primary care with less fragmentation of healthcare delivery, and enables transfer of knowledge to other primary care practices $^{10}$

As CKD shares many commonalities with other chronic diseases (such as diabetes mellitus and cardiovascular disease) in terms of risk factors, prevalence and management approaches, educational initiatives can be collaborative, pooling resources to build a broader and more-comprehensive approach to chronic disease. In pursuing this strategy, lessons can be derived from the experiences of the collaborators, thereby allowing each country to design its own education programmes and delivery approaches adapted to its specific needs and context. An example of this comprehensive approach is the Australian Chronic Disease Prevention Alliance, in which Kidney Health Australia, Cancer Council
Australia, National Heart Foundation of Australia and the Stroke Foundation work effectively together to promote chronic disease prevention, integrated risk assessment, and early detection and management of chronic disease.

An international commission of professional and academic leaders has provided a broad-based set of guidelines to help countries at all levels of economic development to build capacity in terms of education and training in their primary care systems ${ }^{8}$. In addition, the WHO 'Global Strategy on Human Resources for Health: Workforce $2030^{36}$ provides strategic directions for workforce enhancement in primary care that countries can use to develop broad-based training programmes to address several chronic conditions (including CKD), rather than disease-specific approaches.

We have a collective responsibility to bridge the CKD knowledge gap in primary care through more effective strategies of knowledge transfer and quality improvement initiatives (Supplementary Table 1). These approaches have the potential to achieve better kidney health for all while ensuring that the attained training and educational competences align well with socioeconomic, cultural and ethnic realities across countries and settings.

1. Jager, K. J. et al. A single number for advocacy and communicationworldwide more than 850 million individuals have kidney diseases. Kidney Int. 96, 1048-1050 (2019).

2. Wyld, M., Morton, R. L., Hayen, A., Howard, K. \& Webster, A. C. A systematic review and meta-analysis of utility-based quality of life in chronic kidney disease treatments. PLoS Med. 9, e1001307 (2012).

3. GBD Chronic Kidney Disease Collaboration. Global, regional, and national burden of chronic kidney disease, 1990-2017: a systematic analysis for the Global Burden of Disease Study 2017. Lancet 395, 709-733 (2020).

4. Bowe, B., Xie, Y., Xu, E. \& Al-Aly, Z. Kidney outcomes in long COVID. J. Am. Soc. Nephrol. 32, 2851-2862 (2021).

5. Bello, A. K. et al. Global Kidney Health Atlas: A Report by the International Society of Nephrology on the Current State of Organization and Structures for Kidney Care across the Globe, https://www.theisn.org/wp-content/uploads/2021/05/ GKDAtlas_2017_FinalVersion-1.pdf (International Society of Nephrology, 2017)

6. World Health Organization. Global Strategy on Human Resources for Health: Workforce 2030 (Geneva, Switzerland, 2020).

7. Pefanis, A., Botlero, R., Langham, R. G. \& Nelson, C. L. eMAP:CKD electronic diagnosis and management assistance to primary care in chronic kidney disease. Nephrol. Dial. Transplant. 33, 121-128 (2018).

8. Frenk, J. et al. Health professionals for a new century: transforming education to strengthen health systems in an interdependent world. Lancet 376, 1923-1958 (2010).

9. Crowshoe, L. L. et al. Educating for equity care framework: addressing social barriers of Indigenous patients with type 2 diabetes. Can. Fam. Physician 65, 25-33 (2019).

10. Russell, A. W. et al. Clinical outcomes of an integrated primary-secondary model of care for individuals with complex type 2 diabetes: a non-inferiority randomised controlled trial. Diabetologia 62, 41-52 (2019).

\section{Competing interests}

D.W.J. has received consultancy fees, research grants, speaker's honoraria and travel sponsorships from Baxter Healthcare and Fresenius Medical Care, consultancy fees from Astra Zeneca, Bayer and AWAK, speaker's honoraria from $\mathrm{ONO}$ and $\mathrm{BI} \&$ Lilly, and travel sponsorships from Ono and Amgen. He is a current recipient of an Australian National Health and Medical Research Council Leadership Investigator Grant. A.K.B. declares no competing interests.

\section{Supplementary information}

The online version contains supplementary material available at https://doi. org/10.1038/s41581-021-00527-y. 